\section{Lookup table-based inverse model for determining optical properties of turbid media}

\author{
Narasimhan Rajaram, ${ }^{a}$ Tri H. Nguyen, ${ }^{b}$ and \\ James W. Tunnell ${ }^{\mathrm{a}, *}$ \\ ${ }^{a}$ The University of Texas at Austin, Department of \\ Biomedical Engineering, 1 University Station C0800, Austin, \\ TX 78712 \\ ${ }^{\mathrm{b}}$ The University of Texas M.D. Anderson Cancer Center, \\ 1515 Holcombe Blvd., Houston, TX 77030
}

\begin{abstract}
We present a lookup table (LUT)-based inverse model for determining the optical properties of turbid media from steady-state diffuse reflectance spectra that is valid for fiber-based probe geometries with close sourcedetector separations and tissue with low albedo. The lookup table is based solely on experimental measurements of calibration standards. We used tissue-simulating phantoms to validate the accuracy of the LUT inverse model. Our results show excellent agreement between the expected and extracted values of the optical parameters. In addition, the LUT represents a significant improvement in accuracy at short source-detector separations (300 $\mu \mathrm{m})$ and low albedo $(\sim 0.35)$. We also present in vivo data from clinically normal and malignant nonmelanoma skin cancers fit to the LUT-based model. () 2008 Society of PhotoOptical Instrumentation Engineers. [DOI: 10.1117/1.2981797]
\end{abstract}

Keywords: optical properties; reflectance; tissue.

Paper 08115LR received Apr. 7, 2008; revised manuscript received Jun. 26, 2008; accepted for publication Jul. 8, 2008; published online Sep. 22, 2008.

Diffuse optical spectroscopy (DOS) has widely been used to noninvasively characterize tissue optical properties for disease diagnosis. DOS uses optical fiber probes to measure broadband diffuse reflectance spectra from the tissue surface. Collected reflectance spectra are analyzed to extract optical scattering and absorption properties that indicate tissue pathology (i.e., tissue microarchitecture and function). ${ }^{1,2}$ Many current strategies for analyzing diffuse reflectance rely on the solution to the diffusion approximation of the radiative transport equation, ${ }^{3}$ or a modified form. ${ }^{2}$ However, the diffusion approximation is not valid at source-detector separations less than approximately one reduced mean free path $\left[1 /\left(\mu_{\mathrm{s}}^{\prime}\right.\right.$ $\left.\left.+\mu_{\mathrm{a}}\right)\right]$ and in tissues with low albedo $\left[\mu_{\mathrm{s}}^{\prime} /\left(\mu_{\mathrm{s}}^{\prime}+\mu_{\mathrm{a}}\right)<0.9\right]$. In addition, many inverse solutions employing the diffusion approximation are computationally intensive.

Because most cancers originate in the epithelial layer at the tissue surface, DOS systems that sample spectra from the tissue surface are highly desirable. ${ }^{4}$ Also, an important indicator of early cancer, angiogenesis, can lead to significantly higher absorption due to blood at a level comparable to scattering. Unfortunately, the diffusion-approximation-based inverse models are not accurate in many of these regimes. To over-

*Tel: 512-232-2110; E-mail: jtunnell@mail.utexas.edu come this limitation, several recent models based on Monte Carlo $^{5}$ or higher-order approximations ${ }^{6}$ to radiative transport have been developed. In addition, researchers have also developed novel probe strategies and techniques for sampling shallow tissue depths. ${ }^{7-9}$ We present, for the first time to our knowledge, a lookup table (LUT)-based model that is valid at very short source-detector separations $(\sim 300 \mu \mathrm{m})$ as well as highly absorbing media (albedo $\sim 0.35$ ). This method relies solely on a LUT generated from experimental measurements on tissue-simulating phantoms and does not depend on an analytical or computational model of light propagation.

The system we used to collect the diffuse reflectance is described in detail elsewhere. ${ }^{10}$ Briefly, we used a custombuilt clinical spectrometer to collect steady-state, spectrally resolved diffuse reflectance in the wavelength range of 350-700 nm. We used a (i) pulsed xenon flash lamp (L7684, Hamamatsu Photonics) as our light source, (ii) an imaging spectrograph (SP-150, Princeton Instruments) in combination with a 12-bit-cooled CCD (CoolSnap HQ, Photometrics) to collect diffusely reflected light, and (iii) a fiber-optic probe (diameter $=200 \mu \mathrm{m} ; \mathrm{NA}=0.22$ ), where the central fiber illuminated the sample and six surrounding fibers collected the diffusely reflected light. We employed a source-detector separation of $300 \mu \mathrm{m}$ (center-to-center distance). We averaged over three white-light acquisitions to improve the signal-tonoise ratio. The spectral resolution of our system is $\sim 0.78 \mathrm{~nm}$.

We generated the LUT by measuring the functional form of the reflectance using tissue phantoms with known optical properties (calibration set). These phantoms were fabricated using polystyrene microspheres (diameter $=1 \mu \mathrm{m}$; Polysciences) and India ink (Salis International) dissolved in water to simulate scattering and absorption, respectively. We used Mie theory to calculate $\mu_{\mathrm{s}}^{\prime}$ of the tissue phantoms and measured $\mu_{\mathrm{a}}$ of a stock India ink solution using a spectrophotometer (DU 720, Beckman Coulter). We created a matrix (4 $\times 6)$ of 24 tissue phantoms with varying scattering $\left(\mu_{\mathrm{s}}^{\prime}(\lambda)\right.$ $\left.=0.22-7.1 \mathrm{~mm}^{-1}\right)$ and absorption parameters $\left[\mu_{\mathrm{a}}(\lambda)\right.$ $\left.=0-5.33 \mathrm{~mm}^{-1}\right]$, based on previously reported values for tissue. ${ }^{11}$ We found 24 phantoms to be sufficient for overlap in the wavelength-dependent scattering and absorption properties for different phantoms to cover the entire range of the LUT matrix. For example, for consecutive phantoms of increasing $\mu_{\mathrm{s}}^{\prime}$, the lowest $\mu_{\mathrm{s}}^{\prime}$ of each subsequent phantom overlapped with the highest $\mu_{\mathrm{s}}^{\prime}$ of the previous phantom. The probe was placed in contact with the surface of the tissue phantoms, and white-light spectra from the phantoms were recorded [Fig. 1(a)]. Reflectance was calculated by dividing white-light intensity measured from the phantom by whitelight intensity from a reflectance standard (Labsphere). Both measurements were background corrected to account for CCD dark current and ambient light. The measurementmeasurement variation in the diffuse reflectance spectra was $<2 \%$.

The mapping of the spectrally resolved diffuse reflectance $(R)$ on to a unique LUT is shown in Fig. 1. The spectral dependence of $R$ results from the wavelength-dependent optical properties, $\mu_{\mathrm{s}}^{\prime}(\lambda)$ and $\mu_{\mathrm{a}}(\lambda)$. Because $\mu_{\mathrm{s}}^{\prime}(\lambda)$ and $\mu_{\mathrm{a}}(\lambda)$

1083-3668/2008/13(5)/050501/3/\$25.00 @ 2008 SPIE 


\section{JBO LETTERS}

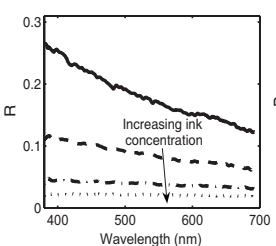

(a) (b)

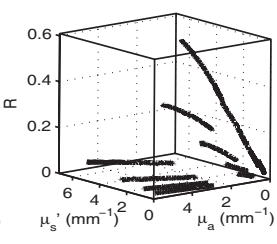

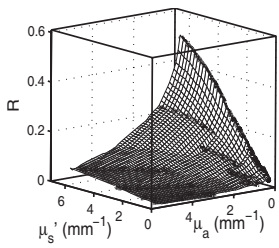

(c)
Fig. 1 (a) Spectrally resolved diffuse reflectance $[R(\lambda)]$ for a subset of tissue phantoms, $\left[\mu_{\mathrm{s}}^{\prime}\left(\lambda_{0}\right)=2 \mathrm{~mm}^{-1}\right.$ and four different ink concentrations corresponding to $\mu_{\mathrm{a}}(\lambda)=0-5.33 \mathrm{~mm}^{-1}$ ], from the calibration set. (b) Diffuse reflectance as a sparse matrix mapped to optical property space, $\left\{R\left[\mu_{\mathrm{s}}^{\prime}(\lambda), \mu_{\mathrm{a}}(\lambda)\right]\right\}$ and (c) the resulting lookup table, $\left[R\left(\mu_{\mathrm{s}}^{\prime}, \mu_{\mathrm{a}}\right)\right]$.

are known for the tissue phantoms, $R$ can be mapped from wavelength space to the two-dimensional optical property space. This mapping creates a sparse matrix [Fig. 1(b)] for $R$. We then interpolated this sparse matrix to a grid of uniformly spaced data points of $\mu_{\mathrm{s}}^{\prime}$ and $\mu_{\mathrm{a}}$ to obtain a LUT for diffuse reflectance [Fig. 1(c)]. The limits of the LUT correspond to the range of $\mu_{\mathrm{s}}^{\prime}$ and $\mu_{\mathrm{a}}$ over which diffuse reflectance spectra were recorded.

Because the LUT is generated with experimental data, using the same absorber to generate the LUT as well as validate it might influence the inverse model while fitting the diffuse reflectance spectra. Therefore, we created a separate matrix $(3 \times 6)$ of 18 tissue phantoms (validation set) with hemoglobin (Sigma-Aldrich) as the absorber.

To fit our diffuse reflectance spectra and extract the optical properties, we implemented a nonlinear optimization fitting routine (average fit time $\sim$ few seconds). We constrained the reduced scattering coefficient to the form $\mu_{\mathrm{s}}^{\prime}(\lambda)$ $=\mu_{\mathrm{s}}^{\prime}\left(\lambda_{0}\right) .\left(\lambda / \lambda_{0}\right)^{-\mathrm{B}}$, where $\lambda_{0}=630 \mathrm{~nm}$. We assumed the absorption in the visible range to be due to oxy- and deoxyhemoglobin. The absorption coefficient was calculated using the absorption cross-sections $\left(\sigma_{\mathrm{Hb}}\right.$ and $\left.\sigma_{\mathrm{HbO}_{2}}\right)$ of these chromophores as $\mu_{\mathrm{a}}(\lambda)=[\mathrm{Hb}]\left(\alpha \sigma_{\mathrm{HbO}_{2}}+(1-\alpha) \sigma_{\mathrm{Hb}}\right)$, where $\alpha$ is the oxygen saturation and $[\mathrm{Hb}]$ is the total hemoglobin concentration. Depending on the type of tissue sampled and the wavelength range of interest, the expression for $\mu_{\mathrm{a}}(\lambda)$ can be modified to include the absorption cross sections of other absorbing chromophores.

The diffuse reflectance spectrum and corresponding fit from a sample validation phantom is shown in Fig. 2(a) demonstrating excellent agreement between the model and the experimental data. Scatter plots of the extracted versus expected

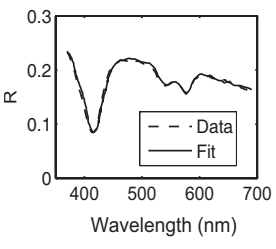

(a)

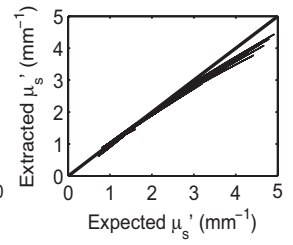

(b)

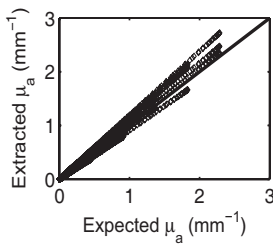

(c)
Fig. 2 (a) Diffuse reflectance spectrum $\left[\mu_{\mathrm{s}}^{\prime}\left(\lambda_{0}\right)=2.49 \mathrm{~mm}^{-1}\right.$ and $[\mathrm{Hb}]=2 \mathrm{mg} / \mathrm{ml}]$ and the LUT-fit from a tissue phantom (validation set). Scatter plot of the known versus measured values of (b) $\mu_{\mathrm{s}}^{\prime}(\lambda)$ and (c) $\mu_{\mathrm{a}}(\lambda)$ for all tissue phantoms. The solid line indicates perfect agreement.

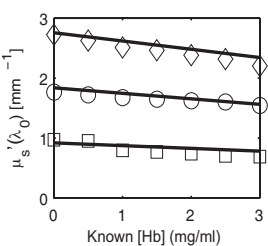

(a)

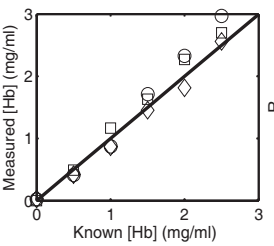

(b)

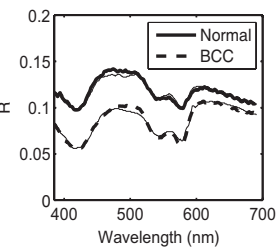

(c)
Fig. 3 (a) and (b) Physical parameters extracted from the LUT inverse model $\left(\mu_{\mathrm{s}}^{\prime}\left(\lambda_{0}\right)=0.91 \mathrm{~mm}^{-1}(\square), 1.83 \mathrm{~mm}^{-1}(\bigcirc)\right.$ and $\left.2.75 \mathrm{~mm}^{-1}(\diamond)\right)$. The solid line indicates perfect agreement. Error bars for these measurements were too small to show $(<2 \%)$. (c) In vivo reflectance spectra from two representative groups: clinically normal and BCC. The thin solid line indicates the model fit.

values of $\mu_{\mathrm{s}}^{\prime}(\lambda)$ [Fig. 2(b)] and $\mu_{\mathrm{a}}(\lambda)$ [Fig. 2(c)] demonstrate a high degree of accuracy in extracting optical properties. The LUT inverse model estimated the reduced scattering and absorption coefficients over a wide range $\left(\mu_{\mathrm{s}}^{\prime}(\lambda)\right.$ $=0.72-4.91 \mathrm{~mm}^{-1}$ and $\left.\mu_{\mathrm{a}}(\lambda)=0-2.29 \mathrm{~mm}^{-1}\right)$ with mean root-mean-square (rms) percent errors (calculated across wavelength and phantoms) of $5.9 \%$ and $11.6 \%$, respectively. These scatter plots show the extracted $\mu_{\mathrm{s}}^{\prime}(\lambda)$ and $\mu_{\mathrm{a}}(\lambda)$ for the entire validation set. Figures 3(a) and 3(b) illustrate extracted physical parameters for each tissue phantom of the validation set. The average errors in estimating $\mu_{\mathrm{s}}^{\prime}\left(\lambda_{0}\right)$ and $[\mathrm{Hb}]$ over the entire validation set were $4.9 \%$ and $9.6 \%$, respectively. We fit the experimental data to the LUT inverse model three times for each phantom, and the variance in the extracted parameters was $<2 \%$. All the experiments were performed immediately after preparation of the phantoms. Therefore, we did not expect the oxygen saturation values to vary, and this was evident in the fits where the values did not vary by $>2 \%$.

We compared the performance of the LUT-based model to a diffusion approximation (DA)-based model described by Farrell et al. ${ }^{3}$ At a source-detector separation of $300 \mu \mathrm{m}$, the LUT model improved the accuracy in recovering scattering at $630 \mathrm{~nm}\left[\mu_{\mathrm{s}}^{\prime}\left(\lambda_{0}\right)\right]$ and hemoglobin concentration $([\mathrm{Hb}])$ by factors of 2.3 and 5.7, respectively. Also, at the lowest value of albedo seen in the validation set (0.35), the LUT model was able to estimate the $\mu_{\mathrm{s}}^{\prime}\left(\lambda_{0}\right)$ and [Hb] with errors of 6.2 and $8 \%$, respectively.

We are currently conducting a clinical feasibility study to determine the sensitivity and specificity of spectral diagnosis for the early detection of skin cancer. Figure 3(c) shows representative spectra from two groups: clinically normal and malignant basal cell carcinoma (BCC). The plot shows good agreement between the predicted (LUT fit) and measured in vivo reflectance. In addition, our preliminary data revealed statistically significant differences in scattering between normal $\left(n=21 ; \quad \mu_{\mathrm{s}}^{\prime}\left(\lambda_{0}\right)=2.1 \pm 0.33 \mathrm{~mm}^{-1}\right)$ and malignant $(n$ $\left.=21 ; \mu_{\mathrm{s}}^{\prime}\left(\lambda_{0}\right)=1.6 \pm 0.3 \mathrm{~mm}^{-1}\right)$ tissue. This difference is consistent with previously reported results for other tissue. ${ }^{2}$

Our analysis indicates that the errors for the LUT-based model are close to $10 \%$ for determining both scattering and absorption. A certain component of the error in our inverse model could arise from the uncertainty in optical properties of the calibration set used to generate the LUT. The experimental error is on the order of a few percent and will propagate 


\section{JBO LETTERS}

through our final inverse solution. Other sources of error include knowledge of bead size and the ink extinction coefficient, presence of electronic noise in the collected reflectance, and fabrication of the tissue phantoms. Minimizing these errors could lead to a significant improvement in the accuracy of the LUT-based model.

Although there have been recent computational models (analytical and numerical Monte Carlo) developed for probes designed for superficial sampling of tissue, more sophisticated probe geometries may be difficult to represent computationally. In addition, unknown system responses (e.g., refractive index mismatch and losses) can be difficult to incorporate into these models. However, an experimental model that is calibrated with the same probe geometry as that used for in vivo measurements should incorporate these factors. Recently, several research groups have developed empirical models calibrated with a set of known optical standards for measuring tissue optical properties. ${ }^{12,13}$ However, these techniques either required a probe geometry with multiple source-detector separations ${ }^{12}$ for developing the model or used a separation well within the diffusion limit. ${ }^{13}$ Our LUT approach extends the bounds of validity imposed by traditional diffusion models to distances very close to the source as well as highly absorbing tissue - an important hallmark of tumors. The model can also possibly be adapted to a wide array of probe geometries. In addition, LUTs can afford a computationally simple and fast approach to inverse solutions.

\section{Acknowledgments}

The funding for this project was provided by the NIH (Grant No. R01 CA132032) and the Wallace H. Coulter Foundation.

\section{References}

1. J. R. Mourant, T. Fuselier, J. Boyer, T. M. Johnson, and I. J. Bigio, "Predictions and measurements of scattering and absorption over broad wavelength ranges in tissue phantoms," Appl. Opt. 36(4), 949-
957 (1997)

2. G. Zonios, L. T. Perelman, V. M. Backman, R. Manoharan, M. Fitzmaurice, J. Van Dam, and M. S. Feld, "Diffuse reflectance spectroscopy of human adenomatous colon polyps in vivo," Appl. Opt. 38(31), 6628-6637 (1999).

3. T. J. Farrell, M. S. Patterson, and B. Wilson, "A diffusion theory model of spatially resolved, steady-state diffuse reflectance for the noninvasive determination of tissue optical properties in vivo," Med. Phys. 19(4), 879-888 (1992).

4. Y. N. Mirabal, S. K. Chang, E. N. Atkinson, A. Malpica, M. Follen, and R. Richards-Kortum, "Reflectance spectroscopy for in vivo detection of cervical pre-cancer," J. Biomed. Opt. 7, 587-594 (2002).

5. G. M. Palmer and N. Ramanujam, "Monte Carlo-based inverse model for calculating tissue optical properties. Part I: Theory and validation on synthetic phantoms," Appl. Opt. 45(5), 1062-1071 (2006).

6. E. L. Hull and T. H. Foster, "Steady-state reflectance spectroscopy in the $\mathrm{P}_{3}$ approximation," J. Opt. Soc. Am. A 18(3), 584-599 (2001).

7. R. Reif, O. A'Amar, and I. J. Bigio, "Analytical model of light reflectance for extraction of the optical properties in small volumes of turbid media," Appl. Opt. 46(29), 7317-7328 (2007).

8. L. Nieman, A. Myakov, J. Aaron, and K. Sokolov, "Optical sectioning using a fiber probe with an angled illumination-collection geometry: Evaluation in engineered tissue phantoms," Appl. Opt. 43(6), 1308-1319 (2004).

9. O. P. Kaspers, H. J. C. M. Sterenborg, and A. Amelink, "Controlling the optical path-length in turbid media using differential path-length spectroscopy: Fiber diameter dependence," Appl. Opt. 47(3), 365371 (2008).

10. N. Rajaram, T. J. Aramil, K. Lee, and J. W. Tunnell, "Clinical instrument for spectral diagnosis of cutaneous malignancy," Proc. SPIE 6848, 68480R (2008).

11. A. J. Welch and M. J. Gemert, "Summary of optical properties," in Optical-Thermal Response of Laser-Irradiated Tissue, Appendix to Chap. 8, Plenum, New York (1995).

12. J. Swartling, J. S. Dam, and S. Andersson-Engels, "Comparison of spatially and temporally resolved diffuse-reflectance measurement systems for determination of biomedical optical properties," Appl. Opt. 42(22), 4612-4620 (2003).

13. P. R. Bargo, S. A. Prahl, T. T. Goodell, R. A. Sleven, G. Koval, G. Blair, and S. L. Jacques, "In vivo determination of optical properties of normal and tumor tissue with white light reflectance and an empirical light transport model during endoscopy," J. Biomed. Opt. 10(3), 034018 (2005). 